\title{
Successful Treatment of Thyroid Carcinoma Invading the Trachea as a Single-Stage Procedure: A Case Report
}

\author{
Kazuhira Endo*, Takayoshi Ueno, Satoru Kondo, Naohiro Wakisaka, Shigeyuki Murono, \\ Tomokazu Yoshizaki \\ Division of Otolaryngology, Graduate School of Medicine, Kanazawa University, Kanazawa, Japan \\ Email: ${ }^{*}$ endok@med.kanazawa-u.ac.jp
}

Received 6 January 2015; accepted 29 January 2015; published 2 February 2015

Copyright (C) 2015 by authors and Scientific Research Publishing Inc.

This work is licensed under the Creative Commons Attribution International License (CC BY).

http://creativecommons.org/licenses/by/4.0/

(c) () Open Access

\begin{abstract}
Objective: Locally advanced thyroid carcinoma is relatively rare; extrathyroid extension, such as to the trachea, is one of the most important prognostic factors. Surgical resection is the mainstay of treatment, and appropriate surgical planning is crucial for obtaining a reasonable prognosis and quality of life of the patients. Case Report: Herein, we report a case of advanced thyroid carcinoma involving the trachea. Total thyroidectomy and window resection of the trachea were performed. The tracheal defect was approximately $2.5 \times 3.0 \mathrm{~cm}$ in size, extending from the first to the third tracheal cartilage, and was reconstructed with auricular cartilage and covered with the sternohyoid muscles of both sides in a single-stage surgical procedure. The tracheal stoma was closed 2 weeks after the surgery. There were no postoperative complications. Conclusion: This reconstruction of the relatively small defect of the trachea provided excellent functional and cosmetic results.
\end{abstract}

\section{Keywords}

Thyroid Carcinoma, Tracheal Reconstruction, Auricular Cartilage, Sternohyoid Muscle, Window Resection

\section{Introduction}

Well-differentiated thyroid carcinoma tends to be potentially curable and has an excellent overall prognosis, with a 10 -year survival rate of over $80 \%$. Although the behavior of this cancer is favorable, $10 \%$ to $15 \%$ of thyroid

\footnotetext{
${ }^{*}$ Corresponding author.
}

How to cite this paper: Endo, K., Ueno, T., Kondo, S., Wakisaka, N., Murono, S. and Yoshizaki, T. (2015) Successful Treatment of Thyroid Carcinoma Invading the Trachea as a Single-Stage Procedure: A Case Report. Case Reports in Clinical Medicine, 4, 50-54. http://dx.doi.org/10.4236/crcm.2015.42012 
carcinomas exhibit aggressive behavior and extrathyroid extension [1]. The commonly involved structures in cases of invasive thyroid carcinoma are the strap muscles, recurrent laryngeal nerve, trachea, esophagus, larynx, and other sites [2] [3]. Invasion of the surrounding structures is considered to be associated with an increased incidence of local recurrence, regional spread and distant metastasis, and a reduced survival rate [3]. Therefore, it is crucial to control local invasion in cases of thyroid carcinoma, which makes treatment relatively difficult. Surgical resection still remains the mainstay of treatment for locally invasive thyroid carcinoma.

In this report, we present a patient with advanced thyroid carcinoma involving the trachea, who was treated by total thyroidectomy and window resection of the trachea. The trachea was reconstructed with a free auricular cartilage graft and the sternohyoid muscles of both sides in a single-stage operation.

\section{Case Report}

A 57-year-old woman presented with a hard mass in her neck that she noticed had been gradually increasing in size in recent months. She had no other symptoms, such as hoarseness, dyspnea or hemoptysis. Her medical history was unremarkable. Flexible fiberoptic laryngoscopy revealed a reddish mass below the vocal cord invading the lumen of the trachea. She was diagnosed with a well-differentiated papillary carcinoma of the thyroid. Computed tomography (CT) (Figure 1) and magnetic resonance imaging (MRI) revealed findings suggestive of carcinoma of the right lobe of the thyroid infiltrating the tracheal wall on the same side and pushing the trachea to the left. The clinical stage was classified as T4NOM0, stage III. Total thyroidectomy accompanied by window resection of the tracheal wall was scheduled. A U-type incision was made with skin flaps to include the platysma muscle. A midline incision was made through the strap muscles. The bilateral sternohyoid muscles were thus separated and preserved. Although the thyroid tumor did not invade the esophagus, deep infiltration of the tracheal wall on the right side was noted. The tumor was removed together with the tumor-infiltrated tracheal wall. The anterolateral tracheal defect was approximately $2.5 \times 3.0 \mathrm{~cm}$ in size, extending from the first to the third tracheal cartilage, involving one-third of the circumference. An auricular cartilage graft with its anterior skin was harvested and inset into the defect of the lateral tracheal wall and sutured (Figure 2(a)). The sternohyoid muscles were used to cover the edge of the tracheal wall defect circumferentially (Figure 2(b)). Orotracheal intubation was changed to tracheostomy intraoperatively before suturing the flaps through the tracheal fistula. The tracheal cannula was kept in place to avoid collapse of the tracheal wall (Figure 3). After surgery, scar formation around the fistula was awaited. The patient was extubated and there was no granulation tissue or stenosis of the tracheal lumen, which was well-epithelialized (Figure 4(a)). The tracheal stoma was closed 2 weeks after the surgery (Figure 4(b)). There were no postoperative complications in either the trachea or the donor site. Neither recurrences nor metastases were found 42 months after the surgery.

\section{Discussion}

Local invasion, such as of the trachea, by thyroid carcinoma can lead to dyspnea, tracheal bleeding and dysphagia, which represent the major causes of the decreased survival of these patients [4]-[6]. There have been several
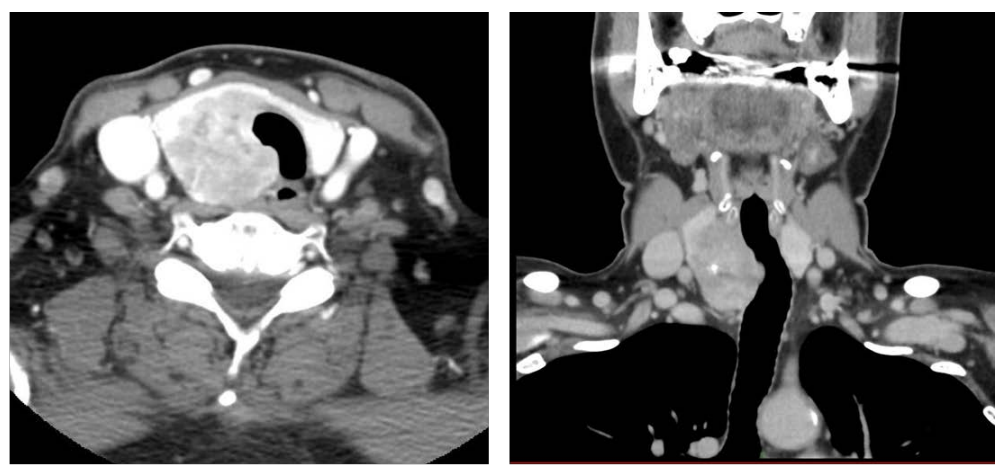

(a)

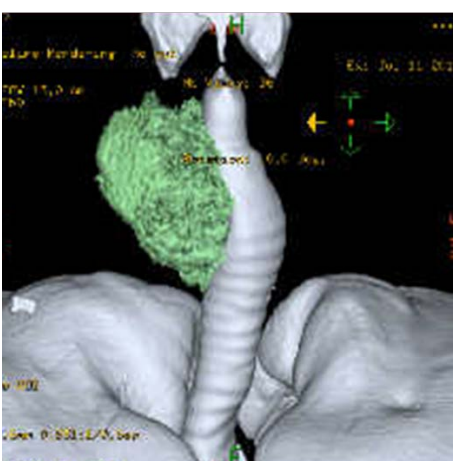

(b)

Figure 1. (a) Cervical computed tomography. Thyroid carcinoma invading the tracheal wall and displacing the trachea laterally; (b) Three-dimensional reconstructed image from fine axial sections shows a shift of the trachea to the right by the tumor. 


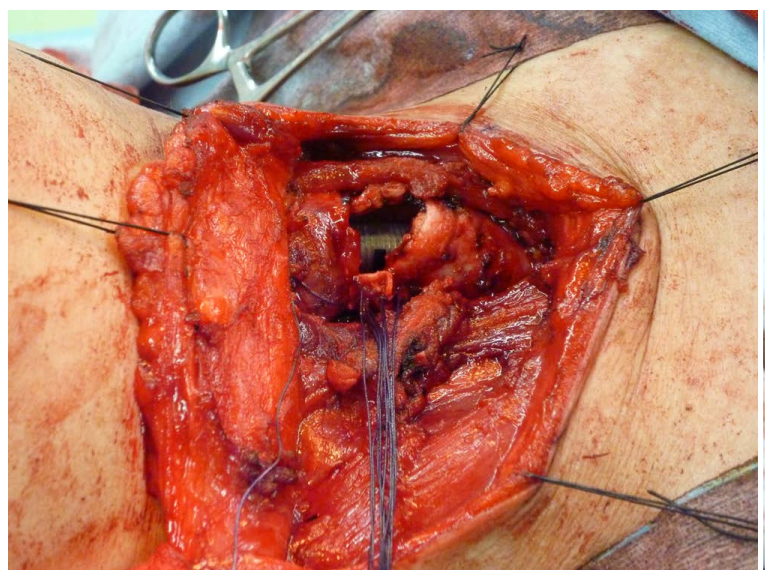

(a)

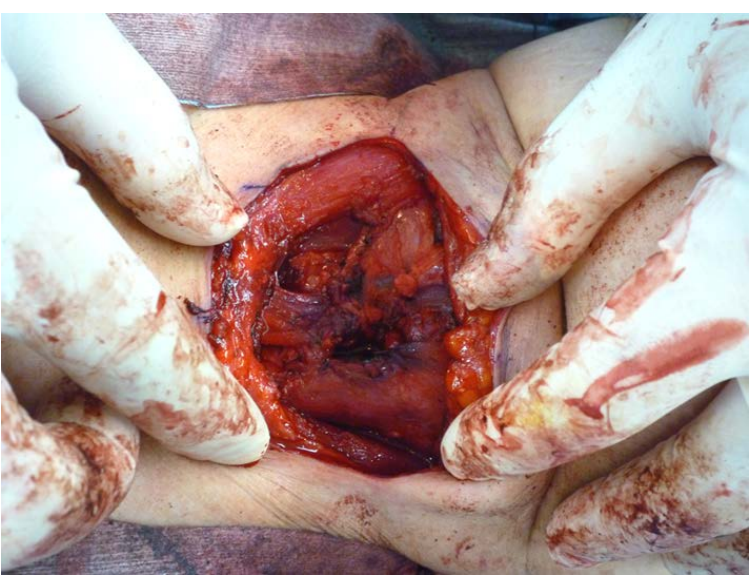

(b)

Figure 2. (a) The tracheal defect was reconstructed with auricular cartilage (arrow); (b) The sternohyoid muscles were used to cover the edge of the tracheal wall defect circumferentially.
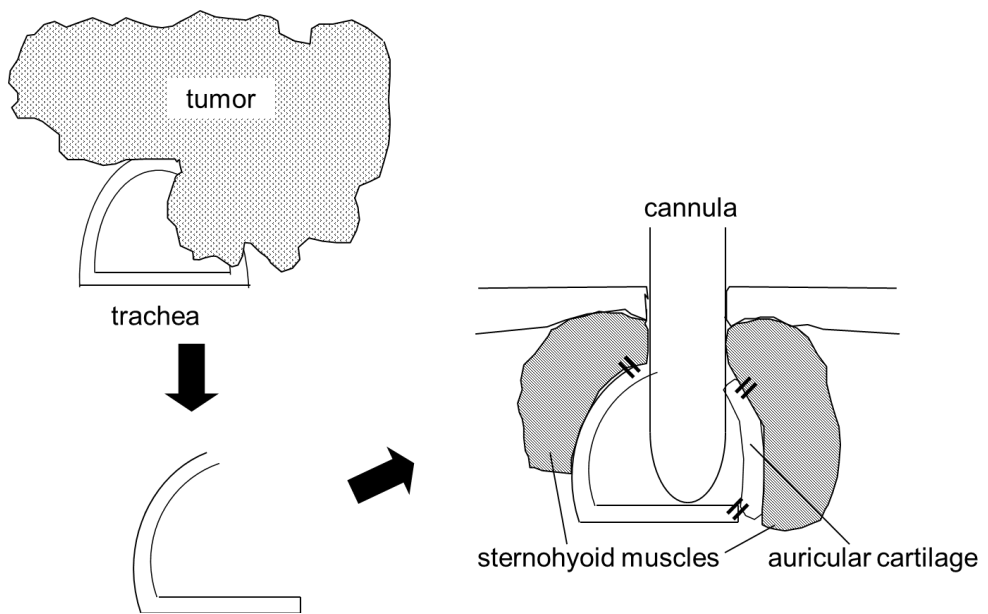

Figure 3. Schema of the operation procedure. The lateral defect of the trachea was reconstructed with auricular cartilage and covered with the sternohyoid muscles of both sides. The anterior defect was converted to a tracheotomy.

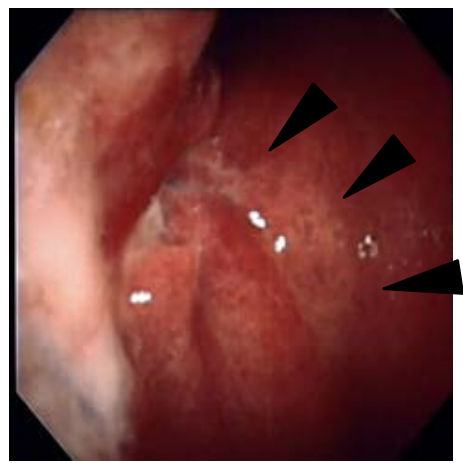

(a)

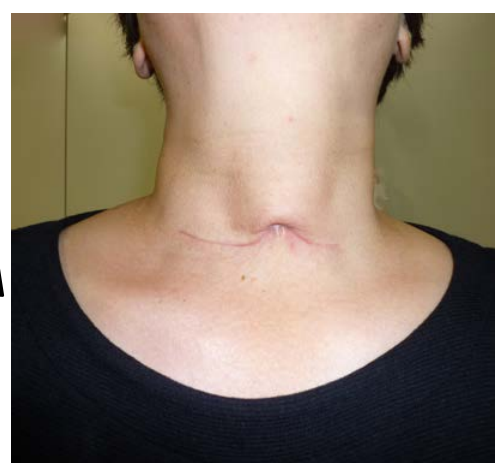

(b)

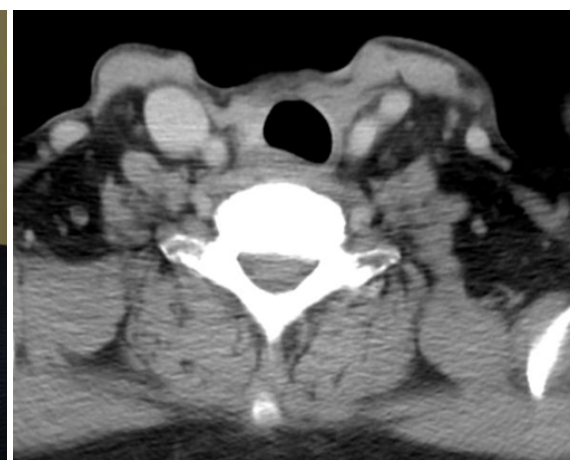

(c)

Figure 4. (a) Two weeks after the operation, the defect of the tracheal wall showed no granulation tissue or stenosis of the lumen, which was well-epithelialized; (b) One month after the surgery, the tracheal stoma was closed; (c) Post-operation CT scan revealed no recurrence or stenosis 6 months after surgery.

reports suggesting that complete resection of thyroid carcinoma invading the trachea can reduce the locoregional recurrence and morbidity rates and improve the long-term survival. 
Various surgical options, on account of the improvement, standardization and more widespread use of reconstructive surgery with microvascular flaps, have been reported in the literature for the management of tracheal invasion by thyroid carcinoma, with no consensus yet on the best approach. The tumor cells invade the tracheal ring cartilages and through the intercartilaginous spaces, the tracheal lumen. Shin et al. devised a pathologic staging system for papillary thyroid carcinoma based on the extent of tracheal invasion (stages I-IV) [7]. McCaffrey has also suggested 5 stages of aerodigestive tract involvement in cases of well-differentiated thyroid carcinoma based on the extent of invasion [8]. However, there is as yet no formal staging system for tracheal invasion in cases of papillary thyroid carcinoma. For appropriate treatment, accurate preoperative diagnosis of tracheal invasion is essential, because presence of symptoms such as hemoptysis, hoarseness, and dyspnea tend to underestimate the depth of airway invasion [9]. Intraluminal tumor inside the trachea is identified by bronchoscopy. Ultrasonography (US), CT, and MRI are also useful to clarify the involvement of adjacent organs; however, these radiological imaging modalities tend to overestimate the extent of invasion [10].

In regard to window resection, there is the problem of reconstruction to avoid an unstable tracheal lumen. Usually, a two-stage surgical procedure is required: first, after resection of the tumor, the tracheal wall and skin are sutured, for the development of a persistent tracheocutaneous fistula "trough". Second, delayed closure of the tracheal defect with a stable framework is performed [11]. Therefore this technique involves long-term treatment and is also not always aesthetically satisfactory because of the need for harvesting a local flap.

In our procedure, the lateral defect of the trachea is reconstructed with auricular cartilage and covered with the sternohyoid muscles of both sides, which are confirmed not to be invaded by the tumor before surgery. The anterior defect is converted to a tracheotomy. Subsequently scar forms around stoma. Reconstruction of the tracheal wall is challenging for the surgeon. There have been previous reports of the use of auricular cartilage or costal cartilage grafts for reconstruction of the tracheal wall [12] [13]. However, these procedures are more than two-step procedures, as mentioned above. Sugenoya et al. have previously reported its usefulness [13] [14]. They also showed that the auricular cartilage and skin are well-vascularized and not easily resorbed. We used an auricular cartilage graft with the anterior skin, because tracheal surface requires to be epithelialized to prevent granulation tissue growth and wound infection, and a stable and supportive framework is necessary to provide an air-tight seal and normal phonation. In our case, there were no complications either at the tracheal wall or at the donor sites.

In the treatment of thyroid carcinoma invading the trachea, one of several options for reconstruction of the trachea can be exercised. We consider that our procedure was less invasive and therefore associated with less surgical stress than previously reported methods. When the resection range of the trachea is small (i.e., extending over 4 rings or less, and half of the circumference or less), closure can be performed without any problems using a local flap [11]. In the case of relatively large defects, however, this method cannot be applied due to the risk of collapse of the tracheal lumen. We consider our method might apply to the reconstruction of tracheal defects up to 4 rings in extent, to avoid collapse or stenosis of the trachea.

\section{Conclusion}

The aim of treatment in cases of locally invasive thyroid carcinoma is to improve the local control and the QOL of the patient while minimizing the morbidity of radical resection, or preserving the functions of adjacent vital structures using conservative procedures. Our modified reconstruction technique for the relatively small defect of the trachea yielded excellent functional and cosmetic results.

\section{References}

[1] Cody III, H.S. and Shah, J.P. (1981) Locally Invasive, Well-Differentiated Thyroid Cancer. 22 Years’ Experience at Memorial Sloan-Kettering Cancer Center. American Journal of Surgery, 142, 480-483. http://dx.doi.org/10.1016/0002-9610(81)90379-2

[2] An, S.Y. and Kim, K.H. (2010) Surgical Management of Locally Advanced Thyroid Cancer. Current Opinion in Otolaryngology Head and Neck Surgery, 18, 119-123. http://dx.doi.org/10.1097/MOO.0b013e3283374d84

[3] Andersen, P.E., Kinsella, J., Loree, T.R., Shaha, A.R. and Shah, J.P. (1995) Differentiated Carcinoma of the Thyroid with Extrathyroidal Extension. American Journal of Surgery, 170, 467-470. http://dx.doi.org/10.1016/S0002-9610(99)80331-6

[4] Nakao, K., Kurozumi, K., Fukushima, S., Nakahara, M., Tsujimoto, M. and Nishida, T. (2001) Merits and Demerits of 
Operative Procedure to the Trachea in Patients with Differentiated Thyroid Cancer. World Journal of Surgery, 25, 723-727. http://dx.doi.org/10.1007/s00268-001-0022-7

[5] Ishihara, T., Kobayashi, K., Kikuchi, K., Kato, R., Kawamura, M. and Ito, K. (1991) Surgical Treatment of Advanced Thyroid Carcinoma Invading the Trachea. Journal of Thoracic and Cardiovascular Surgery, 102, 717-720.

[6] Hammoud, Z.T. and Mathisen, D.J. (2003) Surgical Management of Thyroid Carcinoma Invading the Trachea. Chest Surgery Clinics of North America, 13, 359-367. http://dx.doi.org/10.1016/S1052-3359(03)00018-8

[7] Shin, D.H., Mark, E.J., Suen, H.C. and Grillo, H.C. (1993) Pathologic Staging of Papillary Carcinoma of the Thyroid with Airway Invasion Based on the Anatomic Manner of Extension to the Trachea: A Clinicopathologic Study Based on 22 Patients Who Underwent Thyroidectomy and Airway Resection. Human Pathology, 24, 866-870. http://dx.doi.org/10.1016/0046-8177(93)90136-5

[8] McCaffrey, T.V. and Lipton, R.J. (1990) Thyroid Carcinoma Invading the Upper Aerodigestive System. Laryngoscope, 100, 824-830. http://dx.doi.org/10.1288/00005537-199008000-00006

[9] McCarty, T.M., Kuhn, J.A., Williams Jr., W.L., Ellenhorn, J.D., O’Brien, J.C., Preskitt, J.T., Lieberman, Z.H., Stephens, J., Odom-Maryon, T., Clarke, K.G. and Wagman, L.D. (1997) Surgical Management of Thyroid Cancer Invading the Airway. Annals of Surgical Oncology, 4, 403-408. http://dx.doi.org/10.1007/BF02305553

[10] Lawson, V.G. (1983) The Management of Airway Involvement in Thyroid Tumors. Archives of Otolaryngology, 109, 86-90. http://dx.doi.org/10.1001/archotol.1983.00800160020005

[11] Ebihara, M., Kishimoto, S., Hayashi, R., Miyazaki, M., Shinozaki, T., Daiko, H., Saikawa, M., Sakuraba, M. and Miyamoto, S. (2011) Window Resection of the Trachea and Secondary Reconstruction for Invasion by Differentiated Thyroid Carcinoma. Auris Nasus Larynx, 38, 271-275. http://dx.doi.org/10.1016/j.anl.2010.09.003

[12] Nakahira, M., Nakatani, H., Takeuchi, S., Higashiyama, K. and Fukushima, K. (2006) Safe Reconstruction of a Large Cervico-Mediastinal Tracheal Defect with a Pectoralis Major Myocutaneous Flap and Free Costal Cartilage Grafts. Auris Nasus Larynx, 33, 203-206. http://dx.doi.org/10.1016/j.anl.2005.09.009

[13] Sugenoya, A., Matsuo, K., Asanuma, K., Shingu, K., Shimizu, T., Masuda, H., Kobayashi, S. and Iida, F. (1995) Management of Tracheal Wall Resection for Thyroid Carcinoma by Tracheocutaneous Fenestration and Delayed Closure Using Auricular Cartilage. Head Neck, 17, 339-342. http://dx.doi.org/10.1002/hed.2880170411

[14] Asanuma, K., Itoh, N., Kobayashi, S. and Amano, J. (2000) The Long-Term Outcome of the Cartilage Free Graft to the Tracheal Defect. Head Neck, 22, 95-96. http://dx.doi.org/10.1002/(SICI)1097-0347(200001)22:1<95::AID-HED15>3.0.CO;2-V 
Scientific Research Publishing (SCIRP) is one of the largest Open Access journal publishers. It is currently publishing more than 200 open access, online, peer-reviewed journals covering a wide range of academic disciplines. SCIRP serves the worldwide academic communities and contributes to the progress and application of science with its publication.

Other selected journals from SCIRP are listed as below. Submit your manuscript to us via either submit@scirp.org or Online Submission Portal.
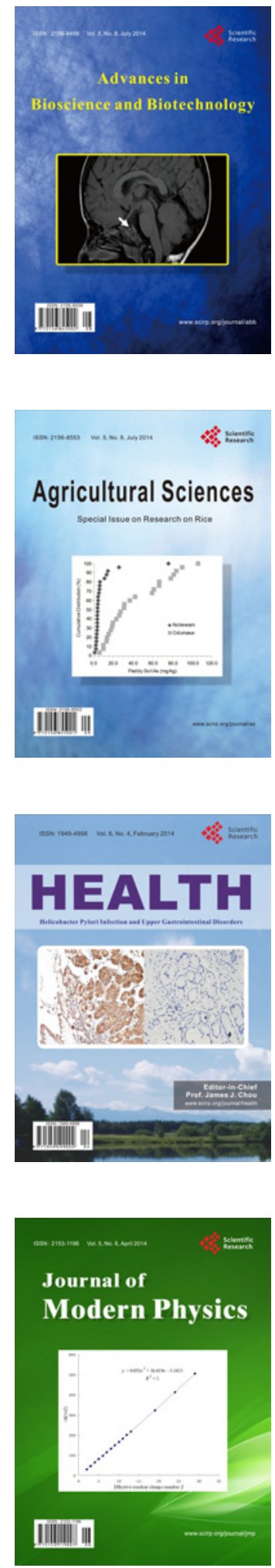
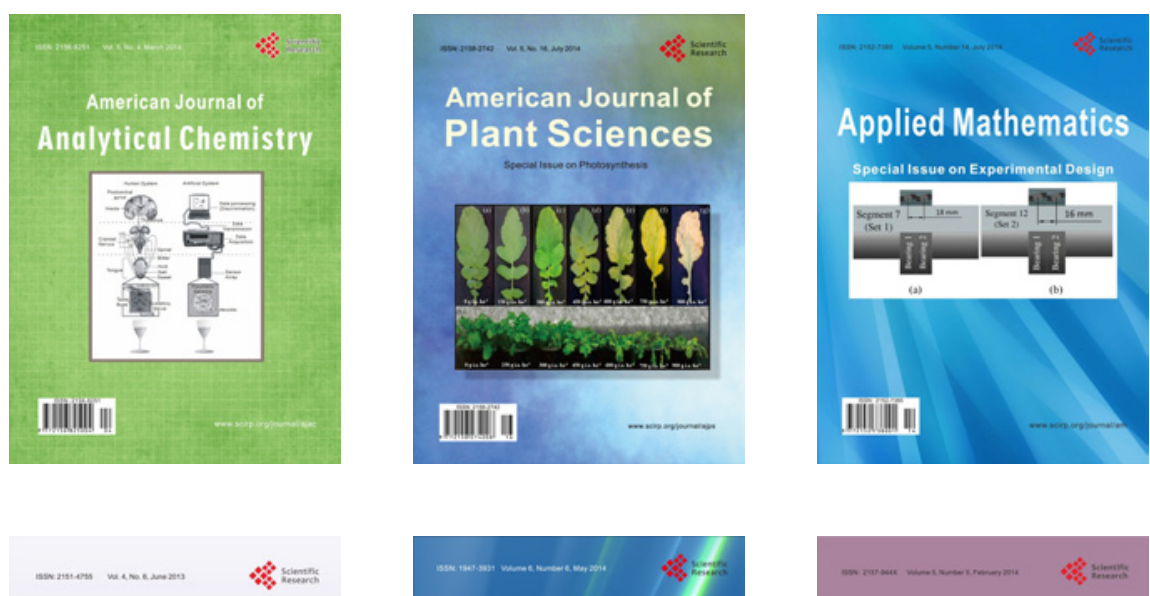

Creative Education
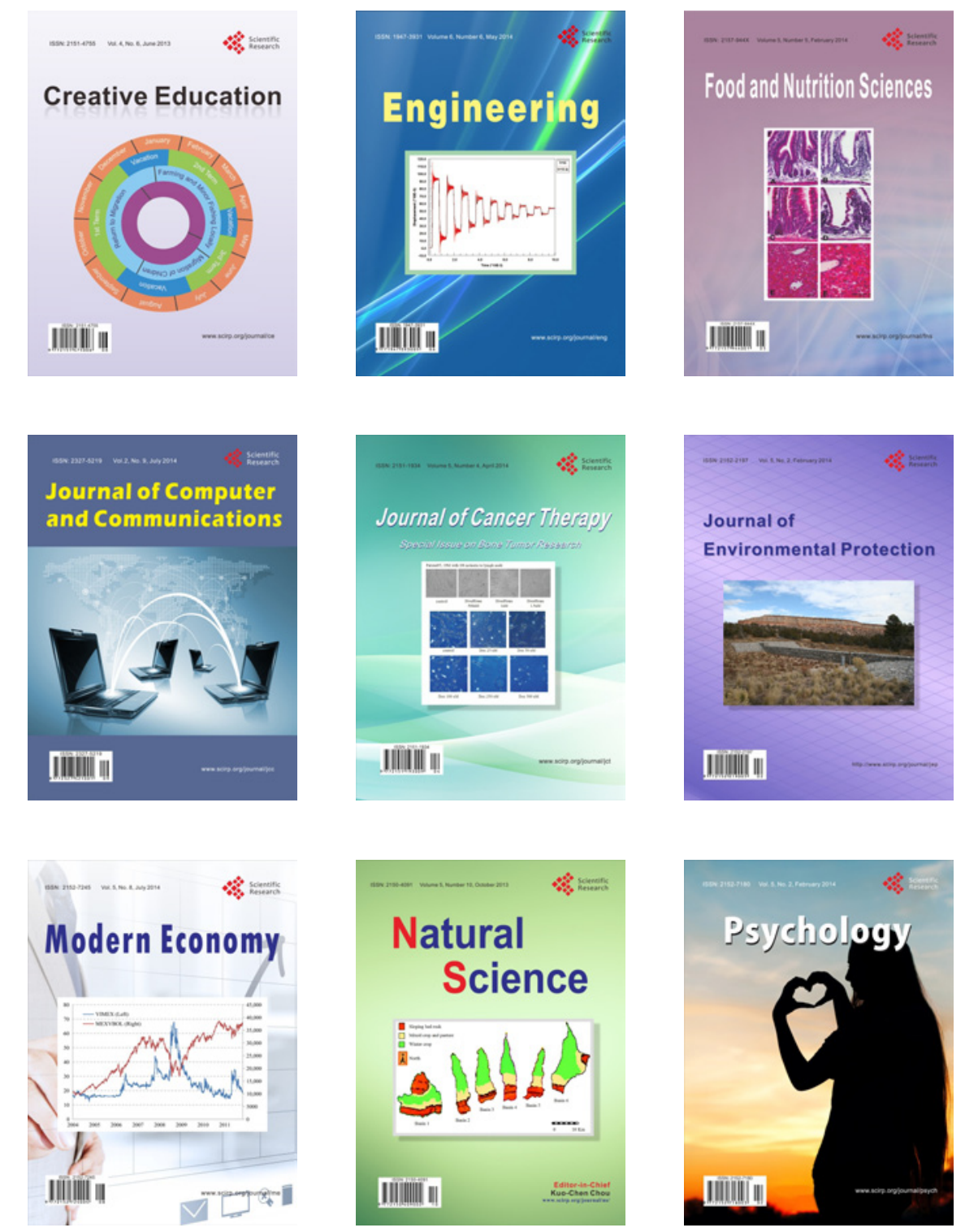